\title{
Analysis on Reasons for Post-Operative DVT of Patients with Lower Limb Fracture in Mining Area and Early Nursing Intervention
}

\author{
Yunjian Liu* \\ Miner Hospital of Xuzhuang, Datun Coal Electricity Company, Pei County, Jiangsu 221611, China
}

\begin{abstract}
Objective: To explore the effectiveness of the early nursing intervention in preventing postoperative deep vein thrombosis (DVT) of patients with lower limb fractures in the mining area. Methods: One hundred (100) patients with lower limb fracture in our hospital were evenly and randomly divided into two groups, namely the observation group and the control group respectively. The early nursing intervention was applied on patients in the observation group, and conventional orthopedic postoperative nursing was applied on patients in the control group. Results: Among 100 patients selected, 15 patients were elderly, accounting for 15\%, 26 patients suffered from the obesity, accounting $26 \%$, there were 25 patients with hypertension, accounting for $25 \%, 16$ patients suffered from diabetes, accounting for $16 \%, 8$ patients suffered from anemia, accounting for $8 \%, 38$ patients had hobbies on the alcohol and tobacco, accounting for $38 \%, 9$ patients had lower limb varicose veins, accounting for $9 \%$, and 35 patients suffered from lower extremity edema, accounting for $35 \%$. The incidence of DVT of patients in the observation group was obviously lower than that of patients in the control group, and the difference was of statistical significance. Conclusions: Early nursing intervention can effectively reduce the incidence of postoperative DVT of patients with lower limb fractures, and it contributes to early recovery of patients.
\end{abstract}

\author{
KEYWORDS \\ Post-operative \\ Lower limb fracture \\ DVT \\ Early nursing intervention
}

\section{Introduction}

The Hospital is located in the mining area, the incidence of fractures during mining showed an obvious hike with intensification of mining mechanization in recent years, especially for lower limb fractures, which were normally caused by direct and (or) indirect force. The incidence of complications also started to go up with the increase fracture patients in mining area, while DVT is one of severe complications of lower limb fractures. The limb swelling and pain, limited mobility and other clinical manifesta-

Copyright $\odot 2015$ Yunjian Liu

doi: $10.18686 /$ jn.v4i3.4

Received: July 2, 2015; Accepted: August 7, 2015; Published online: August 20, 2015

This is an open-access article distributed under the terms of the Creative Commons Attribution Unported License (http://creativecommons.org/ licenses/by-nc/4.0/), which permits unrestricted use, distribution, and reproduction in any medium, provided the original work is properly cited. ${ }^{\star}$ Corresponding author: Miner Hospital of Xuzhuang, Datun Coal Electricity Company, Pei County, Jiangsu province, China 221611. E-mail: liuyunjian008@sina.com tions may be generated when DVT occurs, it may increase and induce deterioration risks of other potential diseases, and it even can cause pulmonary embolism (PTE) and threaten the life of patients [1]. The prophase of DVT lacks clinical manifestation of specificity, increasing the rate of clinically missed diagnosis and the misdiagnosis rate, delaying the treatment, and thereby extending the recovery course of treatment, increasing the patient's pain and economic burden [2]. The author found that early nursing intervention applied on patients with lower limb fracture can effectively prevent the formation of DVT by analysis on 100 patients with lower limb fracture admitted into the Hospital from December 2013 to December 2014. The analysis of results was reported as follows.

\section{Clinical data and methods 2.1. General data}

This group involves 100 patients, including 79 men and 21 women from 37 to 68 with an average at 49 years old. All of them suffered from lower limb fracture, there were 
54 patients with patella fracture, 23 patients with phalangeal fracture, 17 patients with tibiofibular fracture, 4 patients with femoral fracture and 2 patients with femoral neck fracture. Where there were 33 patients with open fracture, 67 patients with closed fracture. Operation methods: 77 patients underwent open reduction and internal fixation, and 23 patients underwent percutaneous fixation. 100 patients were divided into the observation group and the control group with 50 cases in each group. The patients and their families completely understood treatment methods and nursing measures, and they were willing and voluntary to informed consents. The difference in the gender and age were not outstanding $(p>0.05)$ and has the comparability.

\subsection{Nursing method}

Patients in the control group underwent regular orthopaedic postoperative nursing in accordance with Primary Nursing Services for Hospitalized Patients (Trial), Work Specification for Basic Nursing Service, Specifications for Commonly Used Clinical Nursing Technologies and other norms. The patients in the observation group formed nursing interventions to prevent the development of DVT while undergoing routine nursing.

\subsubsection{Postoperative body position nursing}

The patients with lower limb fracture are required to properly put the position and turn over, try best to raise lower limbs by $20^{\circ}$ to $30^{\circ}$ and outreach by $15^{\circ}$ to $30^{\circ}$, the body placement with external plaster fixation should follow principles including the comfort and being conducive to venous return and avoidance of plaster fracture or oppression of local soft tissue. Meanwhile, the monitoring should be intensified, and factors which may result in the circumfluence obstacle of lower limbs should be timely eliminated.

\subsubsection{Health mission}

Properly conduct the risk assessment on the possibility of post-operative patients with DVT, and offer the health education to patients. Improve the awareness of patients on their disease and complications, and eliminate psychological disorders of patients to make them coordinate treatment in more effective manner. In the meantime, help patients drop bad living habits which may induce DVT, such as smoking. Inform patients of the importance of postoperative rational diet for the prevention of DVT, instruct patients take food with low salt, low fat and high protein, ensure daily fluid and nutritional intake to achieve objectives including diluting the blood and preventing the thrombosis.

\subsubsection{Early rehabilitation exercise}

Patients with lower limb fracture are in the state of immobilization or bed rest for a long time, thus increasing the risk of DVT. Some scholars believed that early functional exercise applied on patients could effectively promote the circulation of deep veins at lower limb and reduce the inci- dence of DVT. Early functional exercises include: (1) Take moderate exercises of knee and ankle flexion and extension according to conditions and tolerance states of patients. (2) Guide the patients to master equilong extension and contraction movements of limbs and quadriceps, and guide the patient to independently contact certain times daily. (3) Impose appropriate electrical stimulation on limbs of patients to accelerate the velocity of venous blood flow. (4) It is required to arrange the patient to get off the bed and take movement in order to eliminate whole body high hypercoagulability resulted from long-term immobilization and best rest [3].

\subsubsection{Injection prevention}

The dressing of the wound in accordance with requirements on sterile operation, the body temperature of the patient should be monitored and check if there are signs like the infection, swelling and thermal pain with the wound, and any abnormal situation should be timely reported to the physician-in-charge if any, and the physician should be cooperated to conduct anti-inflammatory treatment in a timely manner.

\subsubsection{Treatment on edema of lower limb}

If the patient suffers from edema, hot and cold compresses should be avoided, as hot compresses may further increase local oxygen consumption and aggravate the disease condition of the patients. While the cold compress may cause further contraction of blood vessels, significantly sharpening the pain, and it is not favorable for patients to set up collateral circulation, and it is appropriate to violently scrub lower limbs and intensely move lower limbs when the patients is undergoing treatment to avoid the fall of thrombus, causing pulmonary embolism. Therefore, the nurse may appropriately raise limbs $20-30 \mathrm{~cm}$ above the lung plane, and closely observe swelling conditions of the patients [4].

\subsection{Statistical analysis}

SPSS 18.0 software is taken to analyze the data collected, in which normal distribution compliant data was subject to one-way ANOVA analysis, and LSD pairwise comparison was taken when statistical significance was satisfied. $p<$ 0.05 prompted there was statistical difference between the two sets of data.

\section{Results}

\subsection{Analysis on DVT risk factors}

Among 100 patients in this study, there were 15 patients with senile ages, accounting for 15\%, 26 patients suffered from obesity, accounting for 26\%, 25 patients had hypotension, accounting for $25 \%, 16$ patients suffered from diabetes, accounting for $16 \%, 8$ patients had anemia, accounting for $8 \%, 38$ patients had alcohol and tobacco hobbies, accounting 38\%, 9 patients had lower-extremity varicose, accounting for $9 \%$, and 35 patients suffered from lower limb edema, accounting for $35 \%$. 


\subsection{Comparison of occurrence of DVT}

Table 1 showed that there was significant statistical difference in the occurrence of DVT of patients in the observation group and that of patients in the control group $(p<0.05)$.

Table 1. Comparison in incidence rate of two groups of DVT.

\begin{tabular}{cccc}
\hline Group name & Cases & $\begin{array}{c}\text { Thrombopoi- } \\
\text { esis }\end{array}$ & $\begin{array}{c}\text { Incidence of } \\
\text { thrombosis (\%) }\end{array}$ \\
\hline Observation group & 50 & 1 & 2 \\
Control group & 50 & 11 & 55 \\
\hline
\end{tabular}

\section{Discussions}

DVT suggests that the blood is abnormally coagulated in the deep vein, and it is determined to be venous reflux disorder of lower extremity. Presently, the incidence of DVT is extremely high, and it has become common vascular disease ranked on second to the coronary heart disease and hypotension according to relevant studies [5]. There are quite a few pathogens causing DVT, and orthopedics surgery for lower limbs is a common factor to induce DVT.

Among the 100 patients in this study, 15 patients were older patients, accounting for 15\%; 26 cases suffered with obesity, accounting for 26\%; 25 patients suffered with hypertension, accounting for 5\%; 16 patients suffered with diabetes, accounting for $16 \%$; 8 patients had anemia, accounting for $8 \%$; 38 patients had alcohol and tobacco addictions, accounting for $38 \%$; 9 patients suffered with varicose veins of lower limbs, accounting for $9 \%$; and 35 cases suffered with lower extremity edema, accounting for 35\%.

Orthopaedic surgeries are likely contributed to generation of high coagulation state of the blood. Selection of anesthesia methods of surgery like spinal anesthesia or general anesthesia, are likely can cause the expansion of surrounding veins, thereby lowering the flow rate of vein. On the other hand, the anesthesia may cause the paralysis of lower limb muscle and the loss of contractile function. While the patient is under immobilization and bed rest for a long time after operation, and lower limb muscles are the state of relaxation, causing sluggish blood flow, thereby inducing the formation of DVT [7].

For causes and risk factors of the formation of the above DTA, comprehensive and systematic nursing should be conducted according to clinical symptoms and history of patients after admission. High-risk groups should be actively educated to improve the cognitive level of patients and their families on DVT. Patients should be guided to master the essentials and follow the doctor's advises. Hypotension, diabetes, anemia and other diseases of the patient should be actively observed and controlled. The patients should be guided to take food with low salt and rich fiber. The constipation and the increase in abdominal pressure should be avoided, drinking of water frequently can help in reducing the blood viscosity, cigarette and alcohol dropping may reduce the irritation on the blood vessel wall [7]. Postoperative function training and anticoagulant therapy can promote local blood circulation, making venous return and preventing slow blood stream resulted from the long time bed rest.

Among DVT risk factors for patients in this group, "postoperative lower limb edema" ranked the highest, which needs more attention. The blood flow of wounded limb must be closely observed after operation, any abnormal situation should be timely handled. Besides, thrombus omen should be timely handled to avoid loss and damage of veins lining [8]. When 100 patients undergoing lower limb fracture surgeries are randomly grouped, two groups of patients were subject to early nursing intervention and traditional nursing. The results showed that patients in the observation group have lower incidence of DVT as compare to control group since they were subjected to postoperative body position nursing, health education, early rehabilitation exercise, prevention of infection, and the nursing intervention measures. The difference in two groups was statistically significance $(p<0.05)$.

To sum up, the application of early nursing intervention on patients who went through the lower limbs fracture surgeries can effectively prevent the formation of DVT, can shorten the treatment cycle, reduce the pain and lighten economic burden of patients. Hence, the author thinks that early nursing intervention is a good method to be promoted as routine nursing for patients after fracture surgeries.

\section{References}

1. Samama MM. A silent killer: exploring the berden of vie in medical patients. VrrE Experts meeting: Belgium. 2004;5:176.

2. Wang SZ, Wang N, Du N. Prevention and nursing of postoperative cerebral venous thrombosis of lower limbs. Journal of Medical Forum. 2011;32(21):189-190.

3. Zhang LP, Ku HG, Pang Y. Influences of health education intervention on deep vein thrombosis of patients undergoing lower limb fracture surgeries. Journal of Qilu Nursing. 2010;(23):4-5.

4. Auqila AM. Deep venous thrombosis. J Cardiovasc Nurs. 2001;15(4):25.

5. Karrholm JG. Uncemented and cemented primary total hip arthroplastty in the Swedish Hip Arthroplasty Register. Acta Orthop. 2010;81:34-41.

6. Li W, Tian YJ, Li JM, et al. Efficacy analysis on air pressure treatment to prevent venous thrombosis of lower limbs after abdominal surgeries. Journal of Practical Diagnosis and Therapy. 2012;26(9):921-922.

7. Wu LJ, Wu ZM, Xu Y. Prevention and nursing on elderly patients with femoral neck fracture combined with deep vein thrombosis. Journal of Guangdong Medical College. 2010;28 (5):586-587.

8. Li Y, Zhou ZG, Zhang HK, et al. Clinical analysis on interventional therapy applied in 56 patients with deep vein thrombosis of lower limb. Journal of Anhui Medicine. 2012;(9): 1164-1165. 